\title{
TARVISION versus TARMED
}

\section{Roland R. Schmoker}

Dr. med. Dr. med. dent., Facharzt für Plastische, Rekonstruktive und Ästhetische Chirurgie, Facharzt für Mund-, Kiefer- und Gesichtschirurgie, Mitglied FMH, Vorstand Tarifunion

Die im TARMED hinterlegten und die in TARVISION neu eingebrachten Eckwerte stehen im Widerstreit zueinander. Sie sollen aus Spezialistensicht bezüglich Qualitätssicherung, Betriebswirtschaftlichkeit, Sicherheit der Tarifanwendung und Tarifautonomie gegeneinander evaluiert werden.

\section{Kritik an TARVISION}

TARVISION hat den Auftrag verfehlt. Das Resultat hat nichts mehr zu tun mit einer Tarifrevision. TARVISION entspricht einem missglückten neuen Tarif. Ein neuer Tarif provoziert Kostenneutralität. In TARVISION fällt auf, dass die Professionalität im strategischen Bereich der Qualitätssicherung und in der Betriebswirtschaftlichkeit fehlt.

Qualitätssicherung: Qualitätssichernde Guidelines gibt es in TARVISION nicht mehr. Patientensicherheit aufgrund einer Zuordnung von qualitativer Dignität, quantitativer Dignität und notwendiger Assistenz fehlt.

Betriebswirtschaftlichkeit: Betriebswirtschaftlichkeit aufgrund eines Vergleichseinkommens in der Lebensarbeitszeit und damit der Dignitätsfaktor fehlen. Der Dignitätsfaktor Operateur/Assistenz ist verkehrt als «Indignitätsfaktor» Assistenz 0,3 eingesetzt. Dies senkt das Assistenzhonorar unter den Lohn einer Hilfskraft. Tarifautonomie: Bezüglich Eckwerten und Bewilligungsverfahren der FMH wird die Tarifautonomie für die Arztleistungen AL missachtet.

\section{Qualitätssicherung}

Die Sicherung der Qualität bildet ein zentrales Anliegen sowohl von FMH wie fmCh. Beide setzen bei Qualitätssicherung auf strukturierte Weiterbildung, z.B. für die Zulassung ausländischer Spezialisten. Die Qualität wird definiert über die qualitative und quantitative Dignität. Diese qualitätssichernden Dignitäten stellen kein gesetzliches Ausschlusskriterium dar. Aufgrund dessen die Leistung nicht erbracht, nicht abgerechnet oder von den Versicherern nicht vergütet werden dürften. Sie widersprechen keineswegs Art. 43 KVG. Die Dignitäten stellen lediglich eine Guideline dar. Die Therapiefreiheit bleibt weiterhin gewährleistet. Dignitäten stellen primär reine Qualitätskriterien dar.
Qualitative Dignität: Die qualitative Dignität definiert die Qualität zwischen den Fachgesellschaften. Die qualitative Dignität bestimmt, welche Fachdisziplinen bezüglich Voraussetzungen für das Erbringen einer Leistung qualifiziert sind. Die qualitative Dignität bleibt an die Leistung gebunden und gehört nicht etwa dem Leistungserbringer.

Quantitative Dignität: Die quantitative Dignität definiert die Qualität innerhalb einer Fachgesellschaft. Die quantitative Dignität gibt für den Leistungserbringer die Mindestanzahl an Jahren strukturierter Weiterbildung für das Erbringen einer bestimmten Leistung vor. Die strukturierte Weiterbildung bezieht sich - im Gegensatz zur Berufserfahrung - auf die spezifischen Leistungen.

Assistenz: Das dritte Qualitätsmerkmal einer Operation ist die Assistenz. Zur Qualitätssicherung gehört unabdingbar, dass für jeden operativen Eingriff die zugehörige Assistenz hinterlegt ist.

Patientensicherheit: Die Patientensicherheit beruht auf der Qualitätssicherung, d.h. auf den im Tarif hinterlegten Qualitätskriterien fachspezifische Zuordnung, strukturierte Weiterbildung in Form der qualitativen und quantitativen Dignitäten und zugeordneter Assistenz.

\section{TARVISION: les paramètres selon les spécialistes}

Dans le projet TARVISION, les paramètres doivent aussi garantir les objectifs poursuivis par les spécialistes. L'assurance-qualité, qui comprend également les valeurs intrinsèques qualitatives et quantitatives, directement inscrites dans le tarif, est garante de la sécurité des patients. Le caractère économique des prestations, qui a pour but de fournir à tous les médecins en exercice un revenu équilibré tout au long de leur pratique professionnelle, se fonde sur le facteur de valeur intrinsèque et sur l'indemnisation des coûts du cabinet. La sécurité d'application du tarif se base sur des prestations à l'acte définies de manière précise. L'autonomie tarifaire doit également offrir aux spécialistes un tarif conforme à leur spécialité. 


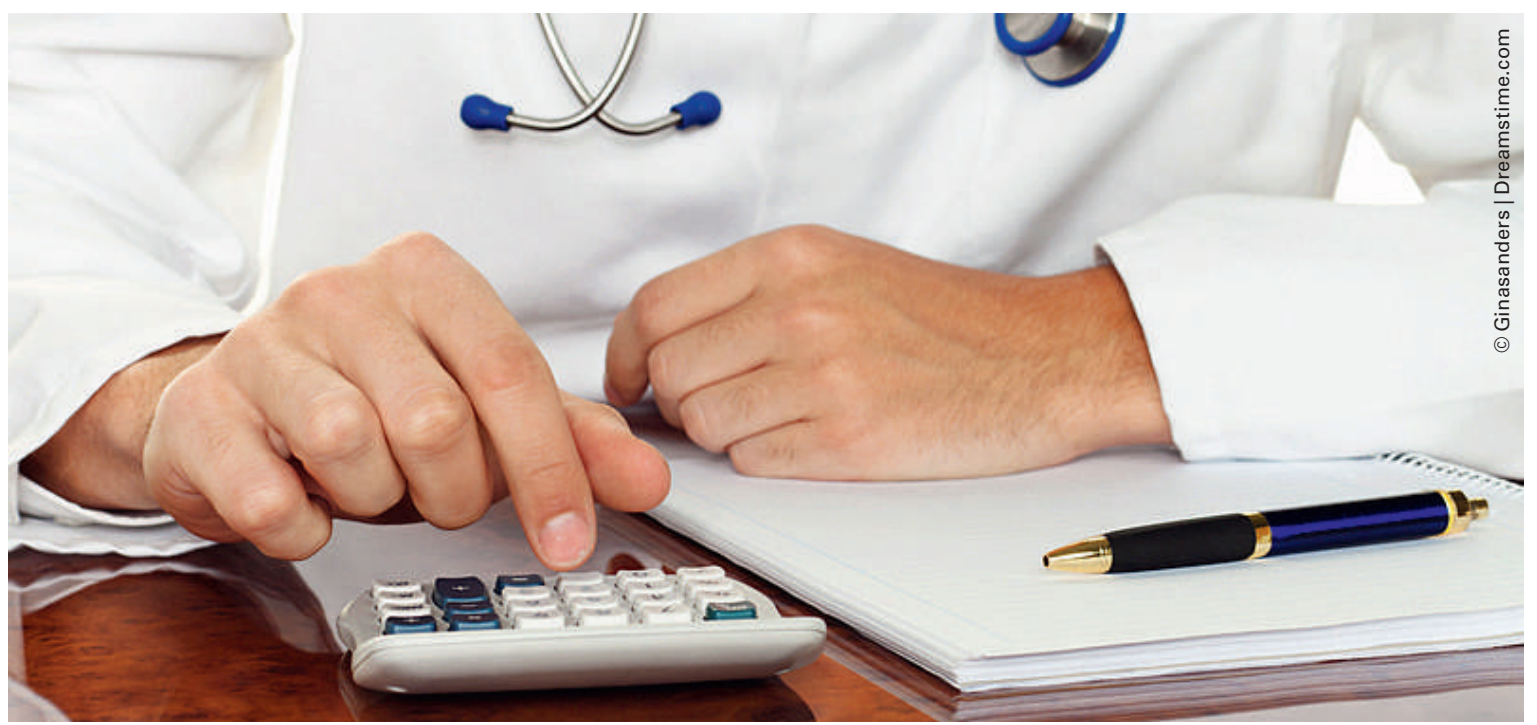

Sicherheit bei der Rechnungskontrolle: Abrechnungen mit Handlungsleistungen sind einfach zu kontrollieren

\section{Betriebswirtschaftlichkeit}

Die Betriebswirtschaftlichkeit soll garantieren, dass ein Leistungserbringer während seiner Lebensarbeitszeit vom Beginn des Medizinstudiums bis zum 65. Altersjahr ein identisches Referenzeinkommen erzielen kann. Die Betriebswirtschaftlichkeit geht also weit über das jährliche Referenzeinkommen hinaus. Die Betriebswirtschaftlichkeit definiert sich über eine ganze Reihe betriebswirtschaftlicher Faktoren.

Handlungs- versus Zeitleistungen: Bisherige Tarife basierten immer vorwiegend auf Handlungsleistungen. Zeitleistungen blieben die Ausnahme für nicht standardisierbare Grundleistungen. Zeitleistungen bei standardisierbaren Operationen würden den unerfahrenen Operateur und das insuffizient arbeitende Operationsteam belohnen. Demgegenüber würden die Erfahrung und die Effizienz benachteiligt. Dies bedingt einen Normierungsfaktor. Der keineswegs auf Handlungsleistungen angewendet werden darf.

Minutage: Die Minutage kann gemessen werden. Die Schwierigkeit besteht allerdings darin, zu entscheiden, wo und bei wem sie als repräsentativ gemessen

\section{Die Dignitäten stellen lediglich eine Guideline dar.}

werden soll. Die Minutage kann mit weniger Abweichungen auch von einem Expertengremium geschätzt werden. Dies hat den Vorteil einer reproduzierbaren Definition. Diese entspricht der Zeit, die ein Operateur nach Einschätzung eines Expertengremiums benötigt, wenn er eine Operation erstmals selbständig durch- führt. Bei der Minutage handelt es sich somit um eine normierte Standardzeit.

Dignitätsfaktor: Die Betriebswirtschaftlichkeit über die ganze Lebensarbeitszeit lässt sich nur mit einem Dignitätsfaktor verwirklichen. Die tarifarische Auswirkung bezieht sich auf die Leistung. Und keinesfalls auf den Leistungserbringer. Wie dies jetzt im IAF vorgese-

\section{TARVISION hat den Auftrag verfehlt.}

hen ist. Ein solcher auf den Leistungserbringer bezogener Dignitätsfaktor wäre bestenfalls VVG-konform, widerspräche aber diametral dem Tarifschutz des KVG. Jede Fachdisziplin hat Mitglieder, die vorwiegend in der Sprechstunde tätig sind. Damit können sie ihre Lebensarbeitszeit nahtlos ausfüllen. Andererseits gibt es Mitglieder, die aufgrund einer längeren Weiterbildung auf operative Tätigkeit spezialisiert sind. Trotz hoher Flexibilität und unregelmässiger Arbeitszeit lässt sich mit Operieren die ganze Lebensarbeitszeit nur unvollständig ausfüllen. Der Dignitätsfaktor soll garantieren, dass mit operativer Tätigkeit, ob Operieren oder Assistieren, ein gleich hohes Lebenseinkommen erzielt werden kann wie mit Sprechstundentätigkeit. Eine FMS-Studie ergab seinerzeit, dass der Höchstanteil an operativer Tätigkeit 15\% der Gesamttätigkeit nicht übersteigt. Massgebend sind keineswegs die sogenannten «Hungerjahre» in der Aus- und Weiterbildung. Vielmehr zählt, ab welchem Lebensalter die Leistung erbracht werden kann? Bis zu welchem Lebensalter ist die Leistung noch zumutbar? Welche berufliche Stellung ist für das Erbringen der Leistung vorgegeben? Erbringung der Leistung als Grundversorger, als Beleg- 
arzt, als Spitalarzt, als Chefarzt? Wie hoch ist das berufliche Risiko in einer entsprechenden Stellung? Was sind die daraus resultierenden Rückstellungen für die Altersvorsorge? Wie stark beeinflusst die Dignität die Prämien der Haftpflichtversicherung? Wie hoch ist der prozentuale Anteil, in welchem die Leistung - bezogen auf die gesamte Lebensarbeitszeit - überhaupt erbracht werden kann? Daraus folgt, dass eine Minute Sprechstundentätigkeit oder eine Minute Assistieren nicht analog abgerechnet werden können wie eine Minute Operieren. Die Vergütungen von einem Operateur und ein bzw. zwei Assistenzen müssten zumindest eine Summe von zwei bzw. drei Grund-Arzt leistungen ergeben. Belegärzte, die sich gegenseitig assistieren, kämen bei TARVISION nur noch auf ein Grundeinkommen von 0,65.

\section{Häufig steht die Auslastung in Konkurrenz zur personellen und materiellen Infrastruktur.}

Produktivität: Die Produktivität spielt sowohl bei der ärztlichen wie bei der technischen Leistung eine fundamentale Rolle. Wenn Leistungen nahtlos über den ganzen Tag aneinandergereiht werden können, resultiert eine hohe Produktivität. Demgegenüber ist die Produktivität beispielsweise von Operationen vergleichsweise niedrig.

Auslastung: Auch der Auslastung von Räumen und Geräten kommt grosse Bedeutung zu. Häufig steht die Auslastung in Konkurrenz zur personellen und materiellen Infrastruktur. Dies kann sich gegenseitig kompensieren. Typisch kommt dies zur Geltung bei der Sparte «Operationssaal». Der Operationssaal eines Spitals ist materiell polyvalent für ganz verschiedene Fachdisziplinen ausgerüstet. Die Auslastung ist sehr hoch. Der Operationssaal einer Praxis ist materiell zielgerichtet auf eine einzelne Fachdisziplin beschränkt. Für die betreffende Fachdisziplin kann der Operationssaal sogar höher qualifiziert sein als der Operationssaal des Spitals. Typisch trifft dies beispielsweise zu für den Fachbereich MKG-Chirurgie. Die Auslastung ist jedoch zwangsläufig gering. Der Unterschied in der Auslastung bewirkt eine identische TL. Diese hängt, unabhängig ob in der Praxis oder im Spital erbracht, nur von der durchgeführten Leistung ab.

Sparten: Bezüglich erbrachter Leistung lassen sich drei Sparten unterscheiden, nämlich der Untersuchungsund Behandlungsraum, der Operationssaal für ambulante Eingriffe (Praxis oder Spital) und der Operationssaal für stationäre Eingriffe. Das sogenannte Clustering von Arztpraxen mit unterschiedlichsten Werten zu einem Durchschnittswert kommt einer Umverteilung gleich. Dabei werden nicht etwa Gewinne verteilt, die angefallen sind, sondern Investitionen und Unkosten, die auf jüngere Ärzte anlässlich der Praxiseröffnung zukommen.

Praxiskostenabgeltung: Die Praxiskostenabgeltung bildet - betriebswirtschaftlich berechtigt - einen massgebenden Anteil des Belegarzthonorars bei am Spital durchgeführten Eingriffen.

\section{Sicherheit der Anwendung}

Die Sicherheit der Tarifanwendung bezieht sich auf unterschiedliche Aspekte.

Sicherheit beim Abrechnen: Höchster Garant für die Sicherheit der Tarifanwendung beim Abrechnen stellen die Handlungsleistungen dar. Der Umfang einer Handlungsleistung ist exakt definiert. Bezeichnet wird die Handlungsleistung direkt vom Operateur. Dies steht im Gegensatz zur Zeitleistung, die nicht exakt definiert ist und vom Operationssaalpersonal angegeben wird. Bei kombinierten Eingriffen wäre eine exakte Zuordnung der sich oft mosaikartig überschneidenden Leistungen selbst für den Operateur nicht möglich. Sobald es nicht nur um eine einzige, sondern um mehrere Einzelleistungen geht, ist ein Abrechnen mit Zeitleistungen völlig unmöglich.

Sicherheit bei der Rechnungskontrolle: Abrechnungen mit Handlungsleistungen sind einfach zu kontrollieren. Die möglichen Zuschlagsleistungen sind vorgegeben. Die Plausibilität einer Kombination von Hauptleistungen lässt sich einfach nachvollziehen. Abrechnungen mit Zeitleistungen sind nicht überprüfbar. Schon bei der Kombination von zwei ungleichen Zeitleistungen ist nicht kontrollierbar, welcher Zeitaufwand der höher bewerteten und welcher der tiefer bewerteten Einzelleistung zukommt.

\section{Der Umfang einer Handlungsleistung ist exakt definiert.}

Sicherheit vor Rückfragen: Die Qualität eines Tarifs bemisst sich unter anderem an der Transparenz bzw. der Unnötigkeit von Rückfragen. Zeitleistungen sind völlig untransparent, insbesondere bei Kombination von Zeitleistungen. Für nähere Angaben sind Rückfragen unausweichlich. Handlungsleistungen sind demgegenüber völlig transparent. Der durchgeführte und abgerechnete Eingriff ist mit der Handlungsleistung in allen Belangen exakt definiert, auch bei einer Kombination von Handlungsleistungen oder Zuschlagsleistungen.

Verhinderung einer Mengenausweitung: Zeitleistungen können bei jeder geringfügigen Änderung im Abrechnungsverhalten zu einer Mengenausweitung führen. 
Dies lässt sich nur durch aufwendige Kontrollen verhindern. Handlungsleistungen sind keiner Änderung im Abrechnungsverhalten unterworfen. Jede Handlungsleistung ist exakt definiert. Die Erbringung beruht auf der medizinischen Indikation eines Fachspezialisten. Indikationskontrollen setzen Handlungsleistungen

\section{Mit Handlungsleistungen lässt sich eine Epidemiologie der durchgeführten Eingriffe begründen.}

voraus. Wirksamster Schutz vor Mengenausweitung beruht auf dem Zusammenfassen von Zeitleistungen $\mathrm{zu}$ einer Handlungsleistung. Paradebeispiel ist der TARMED mit Zusammenfassung der sich überschneidenden Zeitleistungen im Operationssaal vor und nach einem operativen Eingriff. Handlungsleistungen benötigen keinen Normierungsfaktor.

Prävention eines Globalbudgets: Zeitleistungen kommen einem Anreiz für einen Normierungsfaktor bzw. für ein Globalbudget oder für politische Eingriffe in einen Tarif gleich. Umgekehrt setzt die Einführung eines Globalbudgets einen Tarif mit Zeitleistungen voraus. Handlungsleistungen, die betriebswirtschaftlich und auf medizinische Indikationen abgestützt sind, verunmöglichen eine Begründung für die Einführung eines Globalbudgets. Mit Handlungsleistungen lässt sich eine Epidemiologie der durchgeführten Eingriffe begründen. Ebenso können Statistiken über ausgeführte Eingriffe erstellt werden.

Sicherheit vor Rückforderungen: Zeitleistungen stellen wegen der fehlenden Definitionen eine magische Herausforderung für Rückforderungen dar. Dazu wird die Statistik von Zeitleistungen in Form einer gaussschen
Kurve missbraucht. Es werden einfach automatisch $20 \%$ der Zeitleistungen rein spekulativ der Überarztung verdächtigt. Handlungsleistungen können wegen ihrer exakten Definition bestenfalls bezüglich ihrer medizinischen Indikation oder bezüglich der Anspruchshaltung der Patienten in Frage gestellt werden. Bei Handlungsleistungen ist ein lediglich statistisch begründeter spekulativer Pauschalverdacht auf 20\% Überarztung völlig undenkbar. Für eine Rückforderung müsste schon eine eigentliche Falschabrechnung nachweisbar sein, die auf Aufforderung hin nicht korrigiert worden wäre.

\section{Tarifautonomie}

Die Tarifautonomie für die Arztleistung AL muss den Ärzten zugeordnet bleiben. Zur Tarifautonomie innerhalb der Ärzteschaft gehört ein sowohl für die Grundversorger wie für die Spezialisten getrennter Entscheidungsprozess. Es ist undenkbar, dass Grundversorger

\section{Es ist undenkbar, dass Grundversorger} und Spezialisten sich gegenseitig in die Tarifgestaltung dreinreden.

und Spezialisten sich gegenseitig in die Tarifgestaltung dreinreden. Meinungsunterschiede herrschen bei der Dignität zwischen Operateur und Assistenz, beim Dignitätsfaktor bezogen auf die Lebensarbeitszeit, bei der Produktivität beim Operieren und bei der fatalen Auswirkung eines Clusterings auf das Einrichten einer Spezialarztpraxis.

\section{Disclosure statement}

Präsidentenkonferenz KAG Bern, Vorstand Tarifunion und Vorstand BBV+, GPK der SGMKG, Belegarzt 